\title{
Research
}

\section{Optimizing nursing scope of practice within a primary health care context: linking role accountabilities to health outcomes*}

\author{
Jeanne Besner Health Systems and Workforce Research Unit, Calgary Health Region, Calgary, Canada
}

\begin{abstract}
With the increasing focus on collaborative, interprofessional models of service delivery in many parts of the world, it is crucial that nurses be able to demonstrate confidence in the value they add to the health system. As the largest group of health professionals, nurses must serve as change agents in strengthening health systems and influencing the development of appropriate health policy. This requires that nurses have a solid understanding of what is their unique role in contributing to attainment of desired health outcomes in the populations they serve. In this article, the role of nurses in health care is reviewed, with commentary on some areas in which nurses are currently underutilized in health care delivery, whether employed in community or institutional settings. Data from research conducted in Canada suggest that nurses have a tendency to define themselves by the tasks or activities they perform and seem unable to clearly articulate their special role in health care. Strong leadership and a willingness by nurses to re-orient their practice will be required to ensure that the potential for harnessing the knowledge, experience, capabilities and commitment of nurses in advancing health care reform shifts from rhetoric to reality.
\end{abstract}

Key words: nursing role effectiveness; primary health care; role clarity; role accountability

Received: January 2006; accepted: March 2006

\section{Introduction}

This is a time of tremendous opportunity for nurses, regardless of the setting in which they practice, but with that also comes a great deal of responsibility. As the largest group of health professionals, nurses must serve as change agents in strengthening health systems and influencing the development of

\footnotetext{
*This article is based on a symposium presentation delivered at the International Conference on Community Health Nursing Research, Tokyo, 2005.

Address for correspondence: Dr Jeanne Besner, Health Systems and Workforce Research Unit, Calgary Health Region, 10101 Southport Road, Calgary, Alberta, Canada T2W 3N2. Email: Jeanne.Besner@calgaryhealthregion.ca

(C) 2006 Cambridge University Press
}

appropriate health policy in their communities. That requires awareness of the changes that are occurring within the health system and of their potential impact on the advancement of the nursing profession. Most of all, it requires that nurses have a solid understanding of what is their unique role in contributing to attainment of desired health outcomes in the populations they serve.

In this article some of the forces in the external environment are reviewed that are influencing nursing now and no doubt will continue to do so for years to come. The role of nurses in health care is addressed, with commentary on some areas in which nurses are currently underutilized in health care delivery, whether employed in community or institutional settings. Findings from a variety of research 
projects are referenced. In particular, emphasis is placed on the importance of having an explicit understanding of the contribution that nurses make in health care delivery, since it is otherwise extremely difficult to link nursing actions with people's health needs and intended health outcomes. In the absence of such linkages, it is impossible for nurses to document accountability for their practice.

With the increasing focus on collaborative interprofessional models of service delivery in many parts of the world, it is crucial that nurses be able to demonstrate confidence in the value they add to the health system. Research on nursing scope of practice conducted in Alberta and Saskatchewan, Canada has revealed that nurses have a tendency to define themselves by the tasks or activities they perform and seem unable to clearly articulate their special role in health care. The emergence of many new allied health professions, which began in the early 20th century as a subdivision of medicine, appears to have increasingly led to overlap in the activities performed by many health professionals. This overlap in activities has resulted in role confusion, competition among providers, workplace tension, a lack of trust among professionals and a diminishing of professional identity. We can ill afford to continue in this fashion, given the challenges being faced across the world in recruiting and retaining nurses and other health professionals in our health care settings.

\section{The external environment}

In countries like Canada, the UK and many others, recent funding increases in health care have been linked to the expectation that we modernize how services are provided and that we broaden the range of services available to citizens. In an effort to better organize health services around the needs of citizens, many new and innovative programmes have been piloted and are increasingly being incorporated into the health system, particularly in community settings. Drug prescribing (National Health Service, 2001), flexible sigmoidoscopy screening for colorectal cancer (Dobrow, 2005), renewed emphasis on the contribution of nurse practitioners in improving access to health care services (Canadian Nurses Association, 2005; New Zealand Ministry of Health, 2005) and emerging roles in chronic disease management in primary care (Bergman et al., 2000) are just a few examples of opportunities now available to community nurses. All of these potentially increase nurses' capacity to bring a health focus to activities that in the past have often been primarily within the domain of medicine.

Advancements in technologies and constantly changing medical knowledge (College of Physicians of British Columbia, 2005) have meant that many procedures formerly performed by one provider are being delegated to other health professionals (Ontario Hospital Association, 2003). Human resource issues have also led to the call for new interprofessional models of care delivery, more efficient use of resources and a more appropriate skill mix in many health care settings (Government of Alberta, 2000).

Recognizing that health system reform is closely intertwined with the availability of health human resources, many countries have made health workforce issues a major priority. The launch of the Health Workforce Decade (2006-2115) by the World Health Organization testifies to the importance being assigned to appropriate education, planning and management of the health workforce. The draft outline of the World Health Report 2006 also reaffirms that Health for All and primary health care are simply not achievable without an appropriately prepared and deployed health workforce (World Health Organization, 2005).

Therein lies both the challenge and the opportunity for nurses - opportunity because of the inextricable link that exists between nursing and primary health care, but also challenge, because of the difficulty that many nurses seem to have in clearly articulating their role in promoting health for all. As nurses assume more responsibility for procedures that were formerly within the mandate of the medical profession, there is a risk they will be driven even further away from what is their special contribution - that being the promotion of health and wellness - with individuals sick or well.

\section{Nursing and primary health care}

When he was Director General of the World Health Organization, Mahler said: 'If the millions of nurses in a thousand different places articulate the same ideas and convictions about primary health care, and come together as one force, then they could act as a powerhouse for change' (Mahler, 1985). Many would 
nonetheless agree that in the 20 years since Mahler issued that challenge, there has been insufficient global progress in advancing primary health care. Although primary health care reflects the values and principles that have always guided nurses in their practice, there is some evidence that the call to action toward 'health for all' has not resonated with a significant number of nurses. While that is perhaps more understandable for nurses employed in acute care settings, even the practice of community health nurses has tended to become quite medically focused in recent years. If nurses are to play leadership roles in advancing health for all, they must understand the relationship that exists between primary health care and professional nursing practice and re-focus their practice as may be necessary.

\section{What is primary health care?}

The driving force behind the global primary health care movement was the recognition that health is a fundamental human right that is inequitably distributed within, as well as between, the countries of the world. Primary health care reform is aimed at building a sustainable health system focused on improving population health and wellness, by helping individuals and communities accept greater responsibility for their own health, supported by well coordinated and appropriate teams of health providers, whose work is closely aligned with that of other social and economic sectors.

\section{Scope of nursing practice}

These primary health care concepts are totally congruent with the scope of practice of nurses. The International Council of Nurses (ICN) defines the role of nursing as the: 'autonomous and collaborative care of individuals of all ages, families, groups and communities, sick or well and in all settings. Nursing includes the promotion of health, prevention of illness, and the care of ill, disabled and dying people' (ICN, 2005). The object or goal of nursing is what provides the basis for nursing interventions at the individual, family and community levels. The relationship between nursing and health, which is at the core of nursing practice, was clearly articulated by Florence Nightingale, who made little distinction between nursing knowledge and health knowledge, as reflected in her statement that: 'The same laws of health or of nursing, for they are in reality the same, obtain among the well as among the sick' (Nightingale, 1969).

\section{The focus of nursing practice}

The ICN (1996) also notes that effectiveness in nursing practice involves demonstrating that nursing is more than an activity supporting medicine, that it makes a significant contribution to health in its own right. Clearly, while it is true that nurses often carry out medically related functions, they need not adopt a 'medical' perspective while performing their work. In essence, nursing is directed toward helping to create the environments - physical, social and emotional - that are conducive to the attainment of the highest possible level of health and well being for individuals, families and communities. That encompasses much more than simply carrying out a series of procedures or concentrating primarily on illness or disability.

Nursing assessment is the process of making explicit the relationship that exists between such determinants of health as age, medical condition, occupation, socio-economic status and so on, and actual health status. In other words, nursing assessment is directed toward identifying how people's social context (eg, their culture, level of literacy or beliefs about health might influence, either positively or negatively, their response to illness, crisis or life transitions). Synthesizing that information and determining appropriate actions that reflect the complex interplay among those factors is what distinguishes the practice of registered nurses from that of most other health providers.

Nursing intervention on the other hand, is directed at helping people identify and select appropriate strategies that enable them to achieve optimum health and well being, in a spirit of self-reliance and in consideration of their unique circumstances. The strength of nursing and therefore the value added by having nurses in the health system, lies in nurses' ability to identify, interpret and relate, often to other members of the health care team, how the client's unique situation, goals and aspirations may inhibit or facilitate acceptance of, capacity or willingness to follow a prescribed course of action aimed at optimizing health. Nurses need to be far more involved than now appears to be the case in collecting, analyzing, 
using and reporting data related to 'social determinants of health' and in highlighting their influence on people's health and well being. Variability in clients' social context should clearly reflect differences in approach to nursing intervention or health care delivery in general, but all too often, that does not seem to be the case.

\section{Actual practice}

In research conducted with community health nurses working in maternal-child health in Canada, it was found that nurses did not practice in a manner that explicitly reflected nursing or primary health care principles, nor was their practice consistent with generally accepted community health nursing standards (Stewart and Langille, 1995). There was a lack of evidence in their practice of the expected focus on assessment of the broad health needs of clients and their families. Nurses attended to health maintenance and risk reduction, such as managing breastfeeding problems and stressing the importance of immunization against vaccine preventable disease. They did not, however, appear to deal in depth with the substantial challenges faced by some of the clients, particularly single and/or teenage mothers. Nurses described their practice in terms that clearly reflected a narrow focus on physiological or emotional concerns. Although they were generally aware of the client's social situation, they seemed to avoid openly addressing emotional matters or engaging clients in discussing fears or anxieties associated with the burdens some faced in their day-to-day lives. Opportunities to engage clients in working toward the development of improved coping were thus inadvertently missed and sometimes consciously avoided. Client involvement in prioritization of their own issues was generally restricted to the identification and resolution of immediate and primarily medically related concerns, versus longer-term needs or concerns.

This 'medical' or traditional view of health was reinforced in assessment tools and practice guidelines that placed emphasis on detection of physical and psychological problems. It was also reflected in the expectations of clients and other health team members that nurses demonstrate a high degree of technical skill in managing medical concerns. While that is of course important and should not be minimized, it is equally important that expected nursing competencies also encompass knowledge and skills related to the enhancement of health and wellness. If management expectations, agency protocols, professional expertise and problem definition all reflect an orientation toward a bio-medical model of service delivery, nurses will predictably conclude that there are few rewards for adopting a health-promoting style of practice (Besner, 1999).

The same themes that surfaced in research with community health nurses are also evident in research on scope of nursing practice that has just been completed on patient care units in acute care facilities in three health regions in Alberta and Saskatchewan, Canada (Besner et al., 2005). Nurses in these settings commented that their organizations or colleagues do not value their knowledge and skills in promoting health and that heavy workloads and lack of time often prevent them from attending to the cultural, spiritual and psychosocial needs of patients. Yet, it would appear that if the health-promoting role of nurses was more valued in acute care, it would be possible to decrease at least some of the re-admissions to hospital and/or excessive utilization of emergency departments that are now occurring, often within a period of only days or weeks subsequent to discharge from care.

\section{A focus on health and wellness in service delivery}

The role of nurses is critical in strategies aimed at improving population health, providing better access to health care and preventing illness and injury. This was clearly demonstrated in another project in which nurses from the community, as well as practice nurses, partnered with physicians in working toward optimized health for individuals served by a family practice (Besner, 2004). An important element of this primary care project was to create a profile of the population served by the practice, to better understand how a multidisciplinary team of health professionals could effectively deliver primary health care focused services, ensuring provision of the 'right services' by the most appropriate provider. Analysis of utilization data for the practice revealed substantial gaps between the services that were being delivered and what could or should be provided for the 
type of population being served. The elaboration of an explicit framework focusing on the healthpromoting role of nurses as a guide to practice enabled the nurses to assume greater responsibility in providing comprehensive services to clients of the practice. By targeting previously unmet needs in the population (eg initiating routine screening for stress and family violence in the female population), there was greater opportunity to broaden the range of services offered. The public health nurse was instrumental in enabling the practice nurses to fully utilize the knowledge and skills they possessed in assessing the physical, emotional and social needs of clients and in educating people about a wide range of health issues.

Making explicit the health-promoting role of practice nurses allowed them to assume leadership in initiating programmes aimed at re-focusing service delivery toward primary prevention. For example, analysis of demographic data and of risk factors associated with the practice population identified a need to target women's health issues, such as depression, and to concentrate more attention on reduction of cardiovascular risk for the adult population. Women visiting the practice for routine check-ups or episodic health issues are now asked about their emotional well being and receive lifestyle assessment (eg exercise history, alcohol or substance abuse) and support for engaging in activities to enhance their health. Efforts are made to ensure that all adult patients in the practice are routinely screened for cancer and heart disease. The care of patients has shifted from a primary focus on resolution of episodic illnesses to overall management of the total health needs of the population. All members of the health team now work collaboratively to deliver a broad array of health-promoting services. The project has clearly demonstrated the benefits of infusing primary health care principles, such as increased health promotion and team based care, into the delivery of primary care services.

\section{Effective utilization of nursing knowledge}

Strong leadership is required to ensure that the potential for harnessing the knowledge, experience, capabilities and commitment of nurses in advancing the primary health care agenda becomes a reality. If nurses are to play their rightful role in helping to shift our health care system toward a greater emphasis on prevention of disease and injury and promotion of health and wellness, employers, educators, medical colleagues and nurses themselves will have to value nurses' role in promoting health in all settings where they practice. Nurses will only experience frustration if the organizations in which they are employed do not support the holistic, family centred and health related approach to service delivery that is integral to the discipline of nursing.

But nurses themselves will have to lead the way if they want to practice according to the philosophical basis of our discipline. Given current workloads and increasing demands on our time, the only way they can reorient their practice is to let go of some of the activities they are now doing that can perhaps be done just as well by others. Nurses must also assess and document the difference that nursing makes (whether to length of stay, re-admission rates, prevention of adverse events, improvements in patients' knowledge or ability for self-care, etc.) when given the opportunity to focus on improving health and well being, in addition to meeting the 'medically oriented' needs of patients.

\section{Accountability framework}

Quality of nursing care is very much a function of the knowledge base of nurses, of the education and experience they bring to performance of their role, as well as the extent to which they have the time to perform the type and frequency of functions deemed necessary to meet the health needs of people (Institute of Medicine, 2003). The tendency to measure the impact of nursing by reference simply to clients' bio-medical health outcomes has contributed to the problem of 'poorly defined nursing expertise' (Hayward et al., 1993). Consequently, despite the rhetoric of primary health care that characterizes current health reform, the pressures of cost containment are such that the health-promoting role of nurses in achieving improved population health appears to be undervalued by policy makers, politicians, programme developers (Hagan et al., 1995) and, unfortunately, by many nurses as well. If political leaders are to be made accountable for making primary health care the focal point of the health 
system, research relevant to that policy agenda is needed as the basis from which to direct action. The development of an accountability framework that links nurses' scope of practice to their healthpromoting role functions and to the outcomes for which they are held accountable provides a mechanism for identifying actions that can be taken by nurses and others to help strengthen our health system.

\section{How well are we doing?}

Research aimed at eliciting nurses' perceptions of the extent to which they are able to work to 'full scope of practice' suggests that much needs to be done to optimize the roles of nurses. In a study of nursing scope of practice, there was substantial evidence of unmatched expectations between what nurses have been educated to do (ie full scope of practice) and what they perceive they are 'allowed' to do in the practice setting (ie role enactment). Heavy workloads, unavailability of needed resources and the need for more supportive management, were among some of the factors that nurses perceived will need to be addressed, if we are to support them in fully enacting their roles.

Findings from this research also indicate that considerable role overlap and role ambiguity exists not only within nursing, but also across other disciplines. The lack of understanding of differences in professional roles contributes to considerable overlap in task performance and consequent underutilization of the health professional workforce (Besner et al., 2005).

\section{Overcoming challenges}

Optimizing the contribution of all health professionals requires that each provider demonstrate clear areas of expertise that complement, rather than compete with the activities of others (Baranek, 2005). This implies that professionals must have a clear sense of their own roles. Research nonetheless indicates that substantial work is required to clarify roles and responsibilities, improve understanding of the education, competencies and skill base of all members of the health team and provide increased opportunities for meaningful collaboration in delivery of care. The implementation of strategies to address the major health and social needs of individuals, families and communities is where nurses most add value to the health system, but where evidence suggests that nursing knowledge and skills are perhaps most underutilized in our health system at present. If that is going to change, nurses will have to reorient their practice to be more in line with the philosophical underpinnings of the nursing profession. Through their contacts with individuals and families in hospitals, homes, schools, workplaces and other institutional and community settings, nurses have tremend ous opportunities to focus on the creation of environments that support people in attaining their highest possible level of health and wellness. To advance the goal of health for all, nurses will need to make better use of the knowledge they acquire through their day-to-day interactions with people in helping to effect needed social change and improve the quality of our health systems. To shift the focus toward the provision of quality nursing care, we must aim to increase individuals' knowledge of the factors that influence their health and well being and enable them to develop problem-solving skills that will allow them to effectively manage the challenges they face when confronted with illness, disability, disease or other life crises. The health-promoting role functions of nurses that are reflected in their legislated scope of practice must become the frame of reference against which to judge the quality and effectiveness of nursing practice.

\section{Conclusion}

The focus of health systems in many parts of the world is now on finding new ways for health professionals to work differently and in teams. In a rapidly changing health care environment, effective human resource planning is dependent on the understanding and agreement of not only who does what, but also who should perform certain functions and why. Clearly defining and articulating the role of nurses and clarifying what is unique to nursing practice and what is shared with other health professionals poses significant challenge to the profession at this time. Nursing cannot be defined merely by reference to a series of tasks or activities that nurses perform. Rather, we must describe nursing practice in terms of the knowledge and principles that underpin nurses' roles. If nurses are unable to explicate the theoretical basis for their practice, they may find themselves 
unable to clearly articulate what motivates their actions. It is difficult indeed to document accountability for one's practice without an explanatory framework within which to evaluate practice.

Research on the scope of practice in nursing involving community and acute care nurses highlights the importance of clearly articulating what is nursing. This is particularly important at present as all health professionals are being asked to engage in collaborative practice models that will make better use of the knowledge and skills of all members of the health team.

Nurses are experiencing pressure from a number of sources to clarify their scope of practice. Much of the pressure is coming from nurses themselves, who see the need to re-examine their roles in view of changes occurring in the health system. Governments are also calling for a review and modification of existing professional scopes of practice, in order to allow for flexibility in staffing and optimize the use of existing professional resources (Scholes and Vaughan, 2002). Nurses throughout the world must make greater efforts to make explicit the manner in which nursing 'adds value' in the health system, by helping to keep patients safe from harm and enabling individuals, families and communities to achieve their highest possible level of health and well being.

\section{References}

Baranek, P. 2005: A review of scopes of practice of health professions in Canada: a balancing act. Unpublished paper prepared for the Health Council of Canada.

Bergman, J., Delon, S. and Slauenwhite, C. 2000: Multidisciplinary primary care team prevention and management of chronic disease. A report submitted to Alberta Health \& Wellness and the Health Innovation Fund Coordination and Support Team. Calgary, AB. Calgary Health Region.

Besner, J. 1999: A theory-based approach to establishing effectiveness in a public health nursing program. Unpublished doctoral dissertation. University of Alberta.

Besner, J. 2004: Nurses role in primary health care - a call to action. Primary Health Care Research and Development 5, 351-58.

Besner, J., Doran, D., McGillis Hall, L., Giovannetti, P., Girard, F., Hill, W., Morrison, J. and Watson, L. 2005: A systematic approach to maximizing nursing scopes of practice. Retrieved from www.calgaryhealthregion.ca/rinh
Canadian Nurses Association. 2005: Nurse practitioners a viable solution to health care access. Communiqué, September 7, 2005. Ottawa, ON: CNA.

College of Physicians and Surgeons of British Columbia Delegation of a medical act. 2005: Retrieved 12 April 2006 from http://www.cpsbc.ca/cps/physician_resources/ publications/resource_manual/delegationmedicalact

Dobrow, M. 2005: Scope of practice issues for nurse endoscopists. Presentation at the Health Human Resources Summit, Health Council of Canada, June 23, 2005. Toronto, ON.

Government of Alberta. 2000: Alberta statutes and regulations. health professions act. Edmonton: Government of Alberta.

Hagan, L., O'Neill, M. and Dallaire, C. 1995: Linking health promotion and community health nursing: conceptual and practical issues. In Stewart, M.J., editor, Community nursing: promoting Canadian's health. Toronto, ON: W.B. Saunders, Canada, 413-29.

Hayward, S., Ciliska, D., Mitchell, A., Thomas, H., Underwood, J. and Raphael, A. 1993: Evaluation research in public health nursing. A background paper for 'Systematic overviews of the effectiveness of public health nursing interventions (paper 93-3). Hamilton, ON: Quality of Nursing Worklife Research Unit, McMaster University - University of Toronto.

Institute of Medicine. 2003: Keeping patients safe - transforming the work environment of nurses. Washington, DC: The National Academic Press.

International Council of Nurses. 1996: The value of nursing in a changing world. Geneva: International Council of Nurses.

International Council of Nurses. 2005: Retrieved from http:// www.icn.ch/definition.htm

Mahler, H. 1985: Nurses lead the way. The New Zealand Nursing Journal (October), 10-11.

National Health Service. 2001: Maintaining competency in prescribing. An outline framework to help nurse prescribers. Liverpool: National Prescribing Centre.

New Zealand Ministry of Health. 2005: Evolving models of primary health care nursing. New Zealand Ministry of Health. Retrieved from http://www.moh.govt.nz/moh.ns/by+unid.

Nightingale, F. 1969: Notes on nursing. New York: Dover. (Original work published in 1860).

Ontario Hospital Association. 2003: Changing entry-to-practice credentials for regulated health professionals and related employer issues. Finals Report and Recommendations from the HRC ad hoc Credentialism Working Group.

Scholes, J. and Vaughan, B. 2002: Cross-boundary working: implications for the multiprofessional team. Journal of Clinical Nursing 11, 399-408.

Stewart, M.J. and Langille, L.L. 1995: Primary health care principles: core of community health nursing. In Miriam J. Stewart editor, Community nursing: promoting Canadians' health. Toronto, ON: W.B. Saunders, Canada, 62-88.

World Health Organization. 2005: Draft outline: World Health Report 2006. Geneva: World Health Organization. Retrieved from http://www.who.int/hrh/whr06_consultation/en 